VS43-05

\section{Automatic identification of solvatomorphic series in CSD by CrystalCMP}

Jan Rohlicek ${ }^{1}$

1. Department of structure analysis, Institute of Physics AS CR, Prague 8, Czech Republic

email: rohlicek@fzu.cz

In the recent past, several different methods have been published to determine similarities of crystal structures based on the atomic or even molecular positions. These methods can be divided into two basic groups - methods that use so called fingerprints of the crystal structure [1], [2] and methods that directly compare atomic positions [3], [4]. In the first case, an appropriate fingerprint that reflects arrangement of atoms, for example pair distribution function, has to selected. Subsequently, the similarity between two structures is determined by the comparison of their fingerprints. In the second case, it is necessary to find a transformation between the compared structures, then to overlap them and to compare the deviations of the atomic positions. Finding this transformation between two crystal structures is the most difficult task but the great advantage of these methods. The CrystalCMP's [5] method is based, similarly to the xPac [6] and COMPACK [7] methods, on the comparison of a representative molecular cluster where one type of molecule, usually the largest one, is included. During the comparison, the molecular clusters of the individual crystal structures are overlapped and the similarity is calculated as the sum of average deviation of the centers of the overlapping molecules with the average angle between them. Program uses either semi-automatic mode for the comparison, where user has to define a small fragment of the central molecule that will be used for finding the transformation between molecular clusters, or the automatic mode, where the program finds this fragment itself. When the small fragment of the central molecule is known, the comparison can be calculated. After the development of the automatic method, the program was used to compare molecular packing of identical molecules in the whole CSD. First of all, the python script using CSD Python API [8] was used for finding series of crystal structures containing identical molecule in the unit cell. After that, CrystalCMP automatically compared the molecular packing in every series.
References:

[1] M. Valle and A. R. Oganov, Acta Crystallogr. A, vol. 66, no. 5, pp. 507-517, Sep. 2010.

[2] E. L. Willighagen, R. Wehrens, P. Verwer, R. de Gelder, and L. M. C. Buydens, Acta Crystallogr. B, vol. 61, no. 1, pp. 29-36, Feb. 2005.

[3] A. V. Dzyabchenko, Acta Crystallogr. B, vol. 50, no. 4, pp. 414-425, Aug. 1994.

[4] R. Hundt, J. C. Schön, and M. Jansen, J. Appl. Crystallogr., vol. 39, no. 1, pp. 6-16, Feb. 2006.

[5] J. Rohlíček, E. Skořepová, M. Babor, and J. Čejka, J. Appl. Crystallogr., vol. 49, no. 6, pp. 2172-2183, Dec. 2016.

[6] T. Gelbrich, T. L. Threlfall, and M. B. Hursthouse,

CrystEngComm, vol. 14, no. 17, p. 5454, 2012.

[7] J. A. Chisholm and S. Motherwell, J. Appl. Crystallogr., vol. 38, no. 1, pp. 228-231, Feb. 2005.

[8] C. R. Groom, I. J. Bruno, M. P. Lightfoot, and S. C. Ward, Acta Crystallogr. Sect. B Struct. Sci. Cryst. Eng. Mater., vol. 72, no. 2, pp. 171-179, Apr. 2016.

Keywords: similarity, crystal structure, molecular packing, 